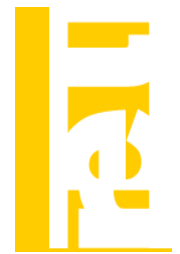

\title{
LA RELACIÓN ENTRE DESEMPLEO Y POBREZA EN LA OBRA DE LUIS TOHARIA
}

\author{
Olga Cantó* Inmaculada García Mainar ${ }^{\dagger}$ \\ * Universidad de Alcalá \\ $\dagger$ Universidad de Zaragoza
}

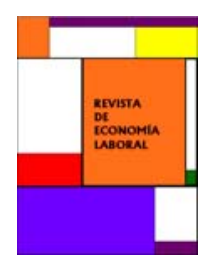

\section{Resumen}

La trayectoria investigadora de Luis Toharia revela una especial preocupación por conocer las implicaciones de las características del mercado de trabajo sobre el bienestar social. Por eso, su investigación busca las claves de la relación entre la situación laboral individual y las condiciones de vida de las familias. Sus principales aportaciones se han centrado en la medición de la capacidad del sistema de protección social para mantener las rentas familiares en situaciones de desempleo duradero, el esclarecimiento de los condicionantes de la relación entre paro, desigualdad, pobreza e inclusión social y la dinámica temporal del empleo y los ingresos familiares.

Palabras clave: pobreza, desempleo, Luis Toharia.

Clasificación JEL: J64, I32.

\begin{abstract}
Luis Toharia's research work reveals a particular concern for investigating the links between the characteristics of the labour market and social welfare. Therefore, his research lines have focused on the key issues that connect individual labour status and household living conditions. His main contributions are centered in measuring to which extent the social protection system maintains household incomes when unemployment is long-term, in identifying the conditions relating unemployment, inequality, poverty and social exclusion and in discovering the connections between employment and household income dynamics.

Key words: poverty, unemployment, Luis Toharia.
\end{abstract}

JEL Classification: J64, I32.

\section{Introducción}

La trayectoria investigadora del profesor Luis Toharia a lo largo de más de tres décadas apunta claramente a una especial preocupación por el efecto de la evolución del mercado de trabajo sobre el bienestar de la sociedad. Esta línea de investigación le llevó a interesarse por un análisis más multidisciplinar de la economía laboral acercándola a otras ramas de la economía que, aunque estrechamente relacionadas con ella, están más centradas en la medición del bienestar económico y en el análisis de las vías para la inclusión social. Así, un número importante de sus trabajos se

(C) Revista de Economía Laboral 
han centrado en la búsqueda de las claves de las estrechas conexiones entre el desempleo y las condiciones de vida de las familias en nuestro país.

Revisando de forma cronológica su amplio elenco de publicaciones podríamos decir que el inicio de esta línea de investigación data de, aproximadamente, finales de los años ochenta, al abordar junto con otros autores un análisis de la relación de las condiciones de vida y trabajo de las familias para un monográfico del Ministerio de Economía y Hacienda véase Muro et al. (1988a). Probablemente, los resultados de este trabajo fueron los que le llevaron a plantearse la necesidad de profundizar más en algunos aspectos de la relación entre desempleo y pobreza en España. En concreto, percibió, en primer lugar, que las implicaciones sociales del desempleo en nuestro país no son tan directas como se podría esperar y, por eso, hizo un gran esfuerzo por tratar de desentrañar las complejas relaciones entre el desempleo distintos miembros del hogar y la posición de la renta familiar en la distribución global de rentas. Como indicaba en uno de sus certeros comentarios sobre el desempleo en nuestro país: "Para entender la naturaleza del desempleo en la España constitucional, es fundamental entender su distribución según la posición ocupada en la familia", Toharia (2003) p.213.

En segundo lugar, desarrolló otros trabajos que le permitieron comparar la capacidad efectiva del sistema de protección social al desempleo en España, pieza clave para relacionar episodios de paro con el bienestar económico familiar, con el existente en otros países europeos y determinar hasta qué punto había diferencias significativas entre ellos en lo que se refiere al grado de mantenimiento de las rentas de las familias cuando alguno de sus miembros está desempleado. En tercer lugar, consideró también fundamental tratar de comprender las relaciones entre desempleo y pobreza a través del análisis dinámico del mercado de trabajo y de las rentas familiares, especialmente a medida que aumentaba la disponibilidad de datos de carácter longitudinal en el contexto europeo.

En este artículo tenemos como objetivo resumir los principales resultados de las contribuciones de Luis Toharia al conocimiento de la relación entre desempleo y pobreza. Para ello, en la primera sección nos centraremos en sistematizar los resultados de sus primeros trabajos en cuanto a las condiciones de vida de las familias y el mercado laboral en España. En la segunda sección nos ocuparemos de comentar los resultados de aquellas aportaciones que analizan el papel fundamental del sistema de protección social al desempleo en la reducción del riesgo de pobreza y, finalmente, en la tercera sección expondremos las conclusiones más importantes de sus artículos centrados en la dinámica del empleo y de las 
rentas familiares. En un último apartado resumimos las conclusiones generales.

\section{Las condiciones de vida de las familias y el mercado de trabajo}

A pesar de que el análisis de la relación del mercado de trabajo con las condiciones de vida de las familias está presente en toda la obra de Luis Toharia, en este artículo vamos a comentar los resultados de una selección de aportaciones científicas que son específicas de este tema y que hemos ordenado de forma cronológica en varios cuadros. Como podemos apreciar en el Cuadro 1, ya en sus primeros años de trabajo investigador, en 1978, Luis se había interesado por el estudio de la economía del bienestar y había publicado un artículo de investigación en la revista Kyklos en el que hacía un comentario teórico sobre las comparaciones interpersonales de bienestar, punto clave en el análisis de corte distributivo de esa disciplina. También en 1984 había escrito junto con Álvaro Espina, su gran amigo Lluis Fina y Felipe Sáez un trabajo sobre la distribución funcional de la renta en España y otro sobre la política de rentas en Cuadernos IMPI. En éste último, se analizaba el Acuerdo Económico y Social de 1984 entre el gobierno, los sindicatos y la patronal sobre salarios, ligándolo a consideraciones sobre la política de rentas franquista y la llevada a cabo por los gobiernos de la transición. Su principal conclusión relativa a las rentas familiares, estrechamente unidas a los salarios, es que durante el periodo franquista la Administración era fuertemente intervencionista en cuanto a los niveles retributivos de las empresas (especialmente durante los años cuarenta y cincuenta del pasado siglo) y, por eso, los acuerdos económicos y sociales entre gobierno, sindicatos y patronal a lo largo de la transición tienen como uno de sus principales objetivos promover la moderación salarial.

En todo caso, es en 1988 cuando Luis escribe, junto con otros autores, lo que podríamos considerar su primera aportación científica centrada en la relación entre desempleo y pobreza: el monográfico realizado en colaboración con Juan Muro, Jose Luis Raymond y Ezequiel Uriel para el Ministerio de Economía y Hacienda (Muro et al., 1988a) que aparece tras otro trabajo realizado en 1987 para la Fundación IESA más centrado en el análisis del mercado de trabajo (Fernández et al., 1988). En este monográfico los autores realizan una la valoración de la nueva Encuesta de Condiciones de Vida y Trabajo (ECVT) para el estudio de las condiciones de vida de las familias y el mercado de trabajo a partir de la información que ofrece sobre el tramo de ingresos al que pertenece cada 
individuo. Es importante destacar que, a juicio de los autores, parece bastante evidente que los datos obtenidos en esta nueva encuesta infravaloran el nivel "real" de ingresos de la población española. A pesar de ello, siendo ésta la única fuente de información disponible en aquel momento, la realización de su análisis resultaba ciertamente interesante.

Uno de los principales resultados de este trabajo, que explota la información de la ECVT referida al cuarto trimestre de 1985, es que el mercado de trabajo español se puede descomponer en tres grandes grupos de trabajadores con diferentes pesos poblacionales: uno con buenas condiciones laborales, buenos salarios y elevada estabilidad laboral (que supondría alrededor de un $50 \%$ de la población ocupada), otro caracterizado por justo lo contrario (un 25\%) y un tercero con características laborales que podríamos clasificar como intermedias (otro $25 \%$ ). Además, los autores concluyen que la probabilidad de estar en un empleo irregular es mayor para los jóvenes, las mujeres y los que están empleados en el sector agrario, mientras que el nivel de estudios o el estado civil no afecta significativamente a esta probabilidad. Otro de los resultados que se obtienen al estimar la probabilidad de tener ingresos por encima de un determinado umbral es que el nivel de estudios, especialmente si son superiores, es el principal determinante de los ingresos individuales de la población española a mediados de los años ochenta. En contraste, otras variables como ser joven, mujer o estar soltero reducen de forma importante la probabilidad de obtener mayores ingresos. Por sectores de actividad, y tomando como referencia el sector servicios, el sector agrícola y la construcción son los que se caracterizan por tener niveles retributivos más bajos que el de referencia, mientras que el sector industrial no se diferencia significativamente del de servicios.

Ligada a esta publicación, ese mismo año de 1988, Luis publica con los mismos autores en la revista Estadística Española - véase Muro et al. (1988b) - un trabajo comparativo de la Encuesta de Población Activa (EPA), centrada en el análisis del mercado de trabajo, y la Encuesta de Condiciones de Vida y Trabajo (ECVT). Esta comparación resultaba muy relevante en aquel momento ya que si ambas encuestas ofrecían resultados similares en cuanto a la tasa de actividad y empleo, la segunda de ellas aparecería como suficientemente fiable para permitir la medición de las condiciones de vida de las familias. Para realizar la comparación de las clasificaciones de la población en relación con la actividad económica en ambas encuestas, los autores utilizan la teoría de grafos. Los resultados apuntan a que la tasa de actividad laboral de la ECVT es muy similar a la que se obtiene en la EPA mientras que se constata que la información de la ECVT permite conocer ciertas actividades marginales del contingente 
de parados que no se consiguen identificar a través de las clasificaciones de la EPA.

La siguiente aportación en este tema, elaborada conjuntamente con Luis Garrido, fue realizada tras la llegada de la fuerte crisis económica de principios de los años noventa a nuestro país y estudia la relación entre desempleo y pobreza desde el punto de vista individual y familiar en términos de la incidencia del desempleo, abarcando desde 1977 hasta 1994. Su objetivo último es explicar una cuestión recurrente en las crisis económicas españolas y observable tanto en las crisis de los ochenta y noventa como en la que sufrimos actualmente: ¿Cómo es posible que la sociedad española pueda soportar una tasa de paro tan alta sin sufrir grandes tensiones sociales? Para responder a esta pregunta el trabajo se centra en la incidencia familiar del paro y utiliza índices de concentración del desempleo para diferentes características como la edad, el sexo, el nivel de estudios y la Comunidad Autónoma. El análisis se realiza a través de índices de desigualdad y de concentración (concretamente una versión del índice de Theil) para algunas de las características de los parados y sus familias. Las conclusiones obtenidas indican que, a pesar del fuerte crecimiento del desempleo durante las dos crisis económicas, una proporción muy elevada de parados ha podido sobrellevar su situación gracias al apoyo prestado por su familia. Por tanto, los autores concluyen que la desigualdad en la carencia económica ha sido un factor clave en explicar que el intenso incremento del desempleo en nuestro país no se haya traducido en fuertes tensiones sociales. La familia ha funcionado como un excelente mecanismo redistribuidor de rentas. En todo caso, los autores indican que, naturalmente, este hecho no implica que ésta deba necesariamente la función de la familia ya que podría argumentarse que en las sociedades desarrolladas existen mecanismos más eficientes de redistribución de la renta.

Debemos señalar aquí que el papel de la familia como "colchón" del alto nivel de desempleo en nuestro país fue un tema al que Luis Toharia dedicó muchos esfuerzos de investigación y también divulgativos, ya que fue tema de muchas de sus conferencias y charlas en las que siempre puso de manifiesto la importancia de la red familiar en las situaciones de crisis económica en nuestro país. En definitiva, sus aportaciones sugieren que la capacidad de soportar altas tasas de desempleo no está tan relacionada con la incidencia del desempleo medida en términos de cuánta población activa está desempleada, sino con su intensidad en términos familiares y, por tanto, la tasa de desempleo individual no es el mejor indicador de las consecuencias sociales del paro que son las que más directamente afectan a la cohesión social en España. 


\section{Pobreza y protección social por desempleo}

En la determinación de la relación entre la pobreza y la situación de desempleo el sistema de protección social juega un papel fundamental. Idealmente, si este sistema cumpliera su principal finalidad se podría evitar que una de las primeras consecuencias de la pérdida del empleo de un miembro del hogar fuera la caída en la pobreza de toda la familia. Como ya indicábamos en la introducción, en la obra de Luis Toharia se pone claramente de manifiesto la importancia que tiene el entorno familiar en la determinación de los efectos del desempleo individual sobre la situación económica del hogar. Para analizar el efecto del desempleo sobre el bienestar de los hogares Luis Toharia aprovecha la estructura de los datos de la EPA, que permite construir la información familiar completa de los individuos entrevistados y pertenecientes al mismo hogar.

En el Cuadro 2 hemos ordenado cronológicamente sus contribuciones en este tema que se pueden dividir, a su vez, en dos temas distintos. El primero de ellos, abordado en el primer trabajo, trata de analizar si es distinta la relación entre desempleo y pobreza en aquellos hogares con desempleados que disfrutan de prestaciones por desempleo frente a aquellos con desempleados que no tienen derecho a ellas o ya no las perciben. El segundo tema, abordado en los dos siguientes artículos, en cambio, describe y analiza las características del sistema de protección por desempleo de nuestro país, comparándolo con el de otros países europeos y evaluando su influencia sobre la equidad y la eficiencia económica.

Cronológicamente, la primera aportación es un capítulo de un libro titulado "Política Social y Estado del Bienestar", elaborado conjuntamente con Inmaculada Cebrián y Luis Garrido. En este trabajo los autores se plantean aportar alguna evidencia acerca de si en la crisis de mediados de los años ochenta junto a las altas tasas de desempleo se produjo también un agravamiento de la duración del paro debido a la bolsa de personas que o no podían salir de esta situación o no tenían incentivos para hacerlo. Para ello, los autores utilizan los datos de la EPA y describen los rasgos característicos de los parados de larga duración, tratando de discernir si la situación es de prevalencia de una bolsa permanente de paro o si, en cambio, hay movilidad dentro del colectivo con individuos que experimentan periodos relativamente cortos de desempleo y varias entradas y salidas de esa situación. La principal conclusión que obtienen es que no se puede decir que el hecho de estar en el desempleo en nuestro país en ese periodo conlleve necesariamente una larga permanencia en el desempleo ya que, efectivamente, se observa que existe una importante movilidad dentro del colectivo de parados. Sin embargo, sí aparecen 
algunos colectivos concreto, relativamente minoritarios, que tienen serios problemas para salir de la situación de desempleo y que, además, carecen de una protección social adecuada.

La segunda aportación sobre este tema se enmarca dentro del segundo grupo de artículos al que nos referíamos antes y cuyo objetivo, en cambio, es describir el sistema español de protección por desempleo. En concreto, analizar la tasa de cobertura y la intensidad protectora del sistema y compararla con la de otros países europeos y realizar un análisis de las características familiares y la percepción de prestaciones de los parados. El artículo describe las características del sistema español de protección por desempleo y su evolución desde sus inicios hasta 1997 poniendo de manifiesto que el diseño del sistema siguió un camino de ida y vuelta caracterizado por una tendencia expansiva hasta 1992 en cuanto a los colectivos cubiertos y una contractiva desde esa fecha, en la que se limita el acceso y la permanencia de algunos grupos.

En segundo lugar, el trabajo se centra en estudiar dos indicadores relevantes del sistema de protección por desempleo: el grado de cobertura y la intensidad de la protección. Respecto del primer indicador, se calcula la proporción de población que disfruta del sistema, utilizando para ello diferentes fuentes de datos y distintas características de los parados. Respecto del segundo, se calcula la tasa de sustitución y se realiza una comparación de la intensidad protectora del sistema español con los existentes en otros países. En tercer lugar, se analizan las características de los perceptores de prestaciones desde dos puntos de vista: la relación con la actividad económica y la situación familiar. En cuarto y último lugar, se aborda una revisión de los estudios españoles que han afrontado el análisis de la influencia de las prestaciones por desempleo en la salida del paro.

La primera parte de este artículo ha constituido, desde su publicación, una excelente guía para conocer de manera profunda las peculiaridades del actual sistema de protección por desempleo español desde sus comienzos (a partir de la Ley Básica de Empleo de 1980) y, en particular, para conocer en detalle las implicaciones de las distintas reformas de los años 1989, 1992 y 1993. La segunda parte, presenta información sobre dos de los elementos fundamentales del sistema de prestaciones y que posteriormente han sido profusamente estudiados en otros artículos de investigación: la tasa de cobertura y la tasa de sustitución. La primera nos informa sobre la cobertura del sistema desde el punto de vista de la cantidad de parados que están protegidos respecto del total mientras que la segunda nos indica la intensidad de dicha protección. 
El análisis que se realiza en este trabajo sobre la tasa de cobertura constituye también un referente de la literatura, ya que incluye una interesante discusión acerca de la manera más adecuada de calcularla, así como diversas valoraciones acerca de los problemas que su cálculo acarrea. En particular, se muestra que los resultados obtenidos utilizando los datos ofrecidos por los registros del entonces Instituto Nacional de Empleo (INEM) ponen de manifiesto que la posible alternativa que suponen éstos frente a los que proporciona la EPA no implica que nos enfrentemos a menos problemas. En definitiva, ambas fuentes estadísticas son interesantes pero ninguna de ellas está exenta de problemas y, en general, no son directamente comparables porque siguen distintos criterios de clasificación. Además, en este trabajo resultan también muy ilustrativos los resultados que se obtienen de la tasa de cobertura para grupos de parados con distintas características personales y diferentes trayectorias laborales.

En cuanto a la intensidad protectora del sistema hay que destacar que este artículo se aborda una interesante discusión sobre el procedimiento de cálculo de la tasa de sustitución y su variación según la trayectoria del trabajador. Sus conclusiones, han sido el punto de partida de varios análisis posteriores (véase Costas y Bel, 1997; Viñals y Jimeno, 1997; Bentolila et al., 2002 y 2004; Cantó y Toharia, 2003 o Toharia et al., 2007). Por otra parte, no menos interesante resulta la comparación de los sistemas de protección de otros países europeos con el español, tanto antes como después de las reformas de 1992 y 1993, que muestra que después de esos dos años de reformas el sistema español resulta ser muy similar al que existe en otros países de nuestro entorno económico.

Finalmente, la tercera parte de este trabajo incluye la caracterización de los perceptores de prestaciones por desempleo y analiza su comportamiento respecto a la búsqueda de empleo. El artículo destaca el cambio que supuso la reforma del sistema de prestaciones que, a partir de 1993, amplió las causas para percibir prestaciones asistenciales (que deja de ser solamente la terminación del periodo de derecho a la prestación contributiva). Como consecuencia de ello, el segundo resultado destacable es que las prestaciones actúan, en muchos casos, más como una renta complementaria que como una renta de subsistencia, pues una parte no desdeñable de perceptores de esta prestación no busca activamente empleo y convive con personas que tienen otros ingresos procedentes del trabajo.

La tercera aportación, elaborada conjuntamente con Olga Cantó en 2003, también corresponde al segundo grupo de artículos, ya que tiene por objetivo evaluar el sistema español de protección por desempleo de principios de este siglo desde la óptica de la eficiencia y la equidad. Para ello, se realizan unas consideraciones teóricas iniciales sobre lo que 
justifica la existencia de un sistema público de protección por desempleo en las economías desarrolladas y sobre los problemas de eficiencia y equidad que plantean estos sistemas. Los autores presentan también las características fundamentales del sistema español de protección por desempleo en el marco del contexto histórico de evolución de estos sistemas en el mundo y evalúan algunos aspectos del mismo desde el punto de vista de la eficiencia y la equidad, utilizando datos de la EPA. Las conclusiones fundamentales que obtienen se resumen en cuatro puntos.

En primer lugar, la cobertura del sistema es una variable generalmente mal medida, ya que las tasas de cobertura que publicaba el antiguo Instituto Nacional de Empleo (INEM), desde 2003 denominado Servicio Público de Empleo Estatal (SEPE), sobreestiman la verdadera dimensión de la cobertura del sistema. Por otra parte, es probable que las tasas de cobertura de la EPA subestimen la cobertura, dada la crecientemente deficiencia en la estimación del número de perceptores de prestaciones que contiene esta encuesta. En tercer lugar, las prestaciones por desempleo son un factor inhibidor de la búsqueda de empleo, ya que un porcentaje sustancial de perceptores de prestaciones declaran no estar buscando empleo y, en consecuencia, la encuesta los clasifica como inactivos y no como parados. Por último, el sistema español de protección por desempleo, en comparación con el existente en los demás países europeos y de la OCDE, se encuentra en un nivel intermedio en lo que se refiere a tres parámetros básicos: el acceso a las prestaciones contributivas, la tasa de sustitución, reposición o "reemplazo" de las prestaciones y la duración potencial de las mismas.

En definitiva, en cuanto al papel del sistema de prestaciones por desempleo en términos de equidad, la obra de Luis Toharia contiene aportaciones en las que se desgrana el funcionamiento del sistema de protección social al desempleo y se analiza la ligazón entre éste y la pobreza. En sus primeros artículos estudia el sistema de una manera más descriptiva aportando alguna evidencia empírica y en los últimos analiza más bien su eficiencia y equidad en comparación con otros sistemas europeos. Para abordar este tema la base de datos que más utilizó fue la EPA, debido a que ésta le permitía el análisis de los individuos en su contexto familiar. En general, sus resultados no encuentran que exista una relación directa entre la pobreza y el desempleo más que para algunos colectivos concretos, poniendo así de manifiesto la importancia de la red familiar para sobrellevar la situación de alto desempleo en las distintas crisis económicas que hemos vivido en el pasado. 


\section{La dinámica del mercado de trabajo y la renta de las familias españolas}

Las posibilidades para realizar estudios sobre la dinámica del mercado de trabajo dependen en buena medida de la disponibilidad de datos longitudinales que permitan abordarlos. Hasta los años noventa del pasado siglo la única posibilidad de análisis longitudinal en el mercado de trabajo español era aprovechar el seguimiento de los hogares encuestados durante seis trimestres consecutivos en la EPA. Esta dimensión longitudinal de la encuesta no proporcionaba un horizonte temporal amplio pero sí permitía, al menos, un seguimiento y ciertos análisis de la trayectoria laboral a lo largo de ese tiempo. Afortunadamente, con la puesta a disposición de los investigadores de las ocho olas sucesivas del Panel de Hogares de la Unión Europea (PHOGUE) durante los años noventa surgió la oportunidad de realizar estudios que habían quedado relegados hasta ese momento por falta de información longitudinal. En García-Serrano et al. (2001), Luis, junto con otros autores, presenta una revisión de todas las posibles aplicaciones del PHOGUE en el estudio de las condiciones de vida de las familias entre las que destacan los análisis sobre los cambios de posición en la trayectoria laboral de los individuos, las transiciones laborales en los distintos países de la Unión Europea, el trabajo a tiempo parcial y la duración de la jornada, los salarios, la pobreza, la discapacidad, la participación y las relaciones sociales.

En el Cuadro 3 se muestran de forma resumida y cronológicamente las publicaciones de Luis Toharia en las que se estudia la dinámica del mercado de trabajo y de rentas familiares. El primero de los trabajos, elaborado conjuntamente con Inmaculada Cebrián y Luis Garrido, ya ha sido comentado en la sección anterior por su análisis de la protección social a los parados. En él se incluye también un estudio dinámico y, por eso, lo incluimos también en esta sección. En este trabajo utilizó, como ya se ha comentado, la dimensión temporal de los datos de la EPA para el estudio de la movilidad de los parados y la obtención de protección en cada momento del tiempo.

El segundo trabajo, realizado conjuntamente con Inmaculada García, toma como punto de partida la relación entre paro y pobreza así como la importancia de la familia en ambas situaciones y utiliza dos fuentes de datos diferentes: el PHOGUE y los datos longitudinales de la EPA. Con los primeros se analiza la pobreza desde el punto de vista de la renta y de las condiciones de vida, y, en la medida de lo posible, de las transiciones entre dos momentos del tiempo, mientras que con los segundos se focaliza en el análisis de la dinámica de desempleo familiar, 
así como en identificar las características que hacen a las familias más proclives a encontrarse en esta situación, a entrar en ella y a tener más dificultades para salir. Los resultados muestran que el desempleo de algún miembro del hogar y, más aún, una situación familiar en la que hay algún parado y ningún ocupado, está claramente relacionado con la pobreza. Sin embargo, muchas de estas familias disfrutan de unas condiciones de vida aceptables y sus problemas económicos no son tan dramáticos como podrían llegar a serlo si el desempleo se convierte en persistente, ya que se observa que la situación de paro suele ser transitoria debido a que casi la mitad de ellas no permanecen en esta situación más de año y medio.

Además, las familias cuya persona de referencia es una mujer con un nivel de estudios bajo son las que muestran más posibilidades de encontrarse en situación de pobreza y también son las tienen más dificultades para salir de ella. Otras características que aumentan la probabilidad de estar en esta situación de baja renta son que el sustentador principal sea joven y que la familia esté conformada por muchas personas, aunque hay que tener en cuenta que esto último también proporciona mayores posibilidades para abandonarla. Finalmente, el nivel de estudios está inversamente relacionado con la probabilidad de ser pobre y, si bien no parece mostrar una influencia clara en la salida de la pobreza, sí incide claramente en la entrada, atemperándola. Se concluye que, en definitiva, paro y pobreza son dos dimensiones que están vinculadas, pero este vínculo está sometido a matizaciones principalmente relacionadas con el papel amortiguador de la familia y la alta movilidad laboral existente en el mercado de trabajo español.

La tercera publicación sobre desempleo y pobreza, elaborada conjuntamente con Carlos García-Serrano y Miguel Ángel Malo, también se ocupa de la relación dinámica entre paro y la pobreza. En ella se muestra que la pérdida de empleo, sin ser un factor desdeñable, no es la única ni la más importante de las causas que hay detrás de la dinámica de la pobreza. Esta conclusión se obtiene a partir de los datos del PHOGUE, que muestran que la persistencia de la pobreza está muy relacionada con la permanencia en el desempleo, aunque, al mismo tiempo, casi tres cuartas partes de los desempleados no habían estado ni transitoria ni continuadamente en la pobreza durante los años de estudio, 1993 y 1994. También se muestra que el desempleo está relacionado con la entrada y salida de la pobreza, especialmente cuando el parado es la persona de referencia del hogar, ya que en ese caso aumenta la probabilidad de entrada en la pobreza y disminuye la de salida. Con todo, la probabilidad de entrada en la pobreza cuando la persona de referencia tiene un empleo 
a tiempo parcial o se encarga de las tareas domésticas es más elevada que cuando esa misma persona está parada.

A juicio de los autores, este carácter complejo de la relación entre paro y pobreza es especialmente útil para discutir la efectividad y el diseño de las políticas de lucha contra esta última, ya que nos muestra, por ejemplo, que una política que incluya el fomento de la salida del desempleo de parados que tienen sus hogares a su cargo puede incidir sensiblemente en la disminución de la pobreza, algo que no sería tan cierto si se dedicara a fomentar la salida del desempleo de cualquier tipo de parado. Por tanto, las políticas específicas dirigidas a colectivos bien definidos son las que tendrían una mayor justificación de acuerdo con los razonamientos expuestos en el artículo; lo mismo se aplicaría a las políticas de prevención, ya que, aunque se comprueba que el paro de la persona de referencia del hogar aumenta la probabilidad de entrada en la pobreza, hay otras situaciones de la persona de referencia (trabajo a tiempo parcial y dedicación exclusiva al trabajo doméstico) que incrementan aún más dicha probabilidad.

La cuarta aportación en este tema, escrita en colaboración con Cecilia Albert, es un capítulo dentro del estudio general sobre el PHOGUE. Los autores se ocupan en este caso de analizar las transiciones laborales en los países de la Unión Europea, con el objetivo principal de mostrar las posibilidades de análisis que presentan los datos de esta encuesta. Para ello, estudian las trayectorias de actividad de los individuos a lo largo de 24 meses de observación, estudiando la evolución longitudinal respecto a la actividad. Definen las transiciones como cualquier cambio de actividad entre los dos momentos del tiempo (finales de 1994 y finales de 1995), distinguiendo entre ocupados, parados e inactivos, y analizan las seis posibles transiciones. También amplían el análisis distinguiendo dentro de los ocupados a los que trabajan más de 15 horas y los posibles cambios de empleo que tienen los trabajadores, que se deducen a partir de la información sobre la antigüedad. Por último, se analiza la movilidad laboral en los diferentes países, con índices agregados de movilidad, y analizando con detalle algunas de las transiciones.

El quinto estudio, realizado con Carlos García-Serrano y Miguel Ángel Malo es un libro que se ocupa de aspectos teóricos y empíricos de la pobreza y utiliza los datos del PHOGUE para la mejora del conocimiento de la exclusión social en Europa, siguiendo uno de los objetivos de la Estrategia Europea de lucha contra la exclusión, definida en los Consejos Europeos de Lisboa y Niza a lo largo del año 2000 y que culminó con la aprobación de la Agenda de Política Social (diciembre 2000). En la parte teórica se realiza una presentación crítica de los métodos habituales de medición de la pobreza y en la parte empírica se realiza un análisis 
transversal de la pobreza a través del indicador de ingresos y otro de privación relativa. Finalmente, se aborda también un análisis longitudinal, comenzando por aspectos teóricos y analizando la evidencia empírica existente. Este estudio resultó novedoso en 2001 ya que fue la primera aportación de investigadores de nuestro país al estudio comparativo de la dinámica de la pobreza en la Unión Europea. En él, además, se destaca la relevancia del concepto de pobreza permanente y las variables socio-demográficas que en ella inciden.

Tal y como indican los autores, la identificación de los pobres y la medición de la amplitud el fenómeno de la pobreza es uno de los problemas fundamentales a los que se enfrentan los investigadores y las instituciones en el área de las políticas públicas. Si bien en el pasado el diseño de las políticas descansaba en la disponibilidad de unos pocos indicadores, desde los años setenta ha habido un resurgimiento del interés por estas cuestiones en los países desarrollados, lo que ha llevado a la aparición de mucha literatura y de innovaciones significativas en el ámbito conceptual y empírico. En concreto, mientras los estudios estáticos sobre pobreza (su intensidad y las características de los individuos o los hogares que son pobres) son relativamente abundantes, los estudios que resaltan los aspectos dinámicos de la pobreza (la entrada y la salida de los individuos o los hogares de la situación de pobreza, su persistencia y los aspectos que influyen en dichos movimientos de entrada y de salida) sin ser escasos pasaron al primer plano de importancia a finales de los años noventa, tanto por la disponibilidad de datos individuales longitudinales sobre rentas y gastos como por el interés en diseñar políticas que atajen el problema de la persistencia de la pobreza familiar. Por esta razón este estudio supone una contribución a la literatura sobre dinámica de la pobreza al realizar una medición de la pobreza permanente en España a mediados de los noventa y compararla con la existente en otros países europeos para el periodo 1994-1996.

Así, tras detectar de forma empírica la relevancia de los pobres permanentes (aquellos cuya renta se encuentra por debajo del 50 por ciento de la media de cada país durante tres años consecutivos), los autores llevan a cabo una descripción de dicho colectivo principalmente en cuanto a su historia laboral y a las características del hogar donde viven. Además, tratan de ofrecer un perfil del mismo utilizando técnicas de análisis multivariante. En todo momento adoptan una perspectiva comparada, en el sentido de buscar si España sigue las mismas pautas de pobreza permanente que el resto de Europa o si, por el contrario, constituye un caso singular.

Los resultados principales que obtienen son los siguientes. En primer lugar, se comprueba que, como era de esperar, la tasa de pobreza 
permanente es menor que la tasa de pobreza corriente. Esto significa que existe un importante grado de movilidad de entrada y salida de la pobreza. En España, un 9,8 por ciento de la población se clasificaría como pobre permanente, mientras que un 75,1 por ciento sería no pobre y el resto, un 15,1 por ciento, experimenta transiciones entre la pobreza y la no pobreza en algunos de los años considerados. Considerando todos los países, la proporción media de pobres permanentes es del 8,3 por ciento y la de los que transitan del 12,7 por ciento, mientras que los nunca pobres alcanzan el 79 por ciento. En segundo lugar, el Reino Unido y varios de los países mediterráneos resultan tener tasas de pobreza anuales y tasas de pobreza permanente que están por encima de la media europea. Sin embargo, la proporción de pobreza permanente es superior a la media Europea en países como Grecia, Portugal e Italia, mientras que en este indicador España se sitúa en la media europea junto con países como el Reino Unido, Bélgica y Francia, lo que indica que existe una mayor movilidad de la pobreza en España que en el resto de países del sur de Europa.

En tercer lugar, en cuanto a la incidencia de la pobreza permanente según la relación con la actividad económica y el historial de desempleo, se construye la variable de "historia laboral" a partir de la información sobre la actividad laboral del individuo en dos periodos distintos: la actualidad y los cinco años anteriores. La incidencia de la pobreza permanente se mide mediante la comparación entre las distribuciones de los pobres permanentes y del total de población según las categorías de situación e historia laboral. Realizando este análisis se detecta la existencia de una gradación en la incidencia de la pobreza persistente en el siguiente sentido: tanto entre los ocupados como entre los parados y entre los inactivos, aquéllos con historias laborales de mucho desempleo están sobrerrepresentados en la distribución de los pobres permanentes en comparación con el total de población, es decir, presentan una incidencia mayor, seguidos de los que tienen poco desempleo y después de aquellos que no lo experimentan en ningún momento. Además, la incidencia de la pobreza permanente es mayor entre los parados que entre los inactivos y los ocupados.

Comparando los resultados de España con el resto de países de la UE, los resultados son parecidos en el sentido de que la mayor incidencia se detecta en personas con historias laborales con muchos episodios de desempleo. Sin embargo, cuando se atiende a la especificidad de cada país, se observa que tanto en Grecia como en Portugal, y sobre todo en España, la incidencia de la pobreza está menos relacionada con el historial de desempleo. Esto podría interpretarse como que en estos países del sur de Europa el riesgo de convertirse en un pobre permanente está mucho menos determinado por la historia laboral. Por tanto, aunque España 
tiene una incidencia de pobreza permanente parecida a otros países, esto esconde importantes diferencias respecto a la protección que ofrece la trayectoria laboral frente a la pobreza permanente, en especial respecto de los países del centro y norte de Europa.

La estimación de un modelo multivariante que permite estimar el efecto aislado de cada una de las variables del análisis sirve a los autores para obtener un perfil de los individuos pobres en un momento determinado, aquellos que lo son de forma permanente y también aquellos que tienen más probabilidades de salir de la pobreza. Los resultados apuntan a que ser mujer, mayor y vivir en un hogar con dependientes son variables que se encuentran estrechamente relacionadas con la pobreza permanente. Sin embargo, las variables que más determinan tanto ser pobre como permanecer un periodo largo en la pobreza son el bajo nivel de estudios y no estar ocupado y, sobre todo, estar desempleado. Las implicaciones más importantes de todos estos resultados, a juicio de los autores, es que el objetivo de una política contra la pobreza permanente debería tener dos pilares: articularse más en torno a las familias que en torno a los individuos $\mathrm{y}$, en segundo lugar, incentivar y favorecer el empleo. Los resultados obtenidos en España en cuanto a la mayor privación de los que realizan actividades por cuenta propia plantea la posibilidad de estudiar de forma más detallada la situación de los autónomos, que podrían estar afectados por privaciones no relacionadas tanto con limitaciones de ingresos como con problemas relacionados con su variabilidad.

La última aportación en este tema fue elaborada por varios coautores de Luis Toharia y constituye el quinto capítulo del libro publicado por el Ministerio de Trabajo y Asuntos Sociales titulado "Empleo e Inclusión Social". En este trabajo se lleva a cabo un estudio dinámico de la pobreza desde 1994 hasta 2001, utilizando datos del PHOGUE. El trabajo tiene como principal objetivo diferenciar la pobreza persistente de la pobreza transitoria y analizar las diferentes características de los individuos que experimentan ambos tipos de pobreza con objeto de comprobar hasta qué punto son similares. Los resultados muestran que la pobreza persistente y la ocasional obedecen a procesos parecidos y, por consiguiente, las políticas de actuación deberían ser similares, si bien matizando la intensidad de las políticas en función de algunas variables ya que se observa que el grado de influencia de variables relevantes en ambos casos es ciertamente diferente.

En definitiva, la principal aportación de todos estos trabajos de Luis Toharia es la de poner de manifiesto la importancia de los estudios dinámicos que combinan el análisis del mercado de trabajo y de las rentas familiares y que aprovechan la información de bases de datos europeas 
disponibles. Todos ellos muestran las enormes posibilidades de una gran base de datos homogénea para todos los países de la Unión Europea como el PHOGUE que realiza un seguimiento de los hogares año a año y proporciona información sobre rentas y variables del entorno laboral de la población. Además, estos trabajos subrayan que para el estudio de la relación entre desempleo y pobreza es importante tener en cuenta la dimensión longitudinal de la pobreza y el empleo, ya que ambas situaciones, aunque estrechamente relacionadas, están condicionadas por la movilidad laboral y el papel de la familia que tienen efectos dinámicos que sólo pueden ser capturados teniendo en cuenta la movilidad en las situaciones de empleo y desempleo y en las rentas familiares.

\section{Conclusiones}

En este artículo hemos querido subrayar que el trabajo de investigación del profesor Luis Toharia revela una especial preocupación por conocer las implicaciones que tienen las características del mercado de trabajo sobre el bienestar social. De hecho, a lo largo de toda su trayectoria investigadora realizó una intensa búsqueda de las claves de las estrechas conexiones entre el mercado de trabajo y las condiciones de vida de las familias. Sus principales aportaciones en esta línea se han centrado en la medición de la capacidad del sistema de protección social para mantener las rentas familiares en situaciones de desempleo duradero, el esclarecimiento de los condicionantes de la relación entre paro, desigualdad, pobreza e inclusión social y, a partir de la disponibilidad de datos de carácter longitudinal en el contexto europeo, la descripción de la dinámica temporal del empleo y los ingresos familiares. A lo largo de todo este artículo hemos resumido las principales conclusiones de sus trabajos sobre la relación entre desempleo y pobreza. Para terminar queremos señalar que, desde nuestro punto de vista, su aportación más importante en esta línea de investigación ha sido subrayar la gran relevancia que tiene en España el apoyo económico familiar y la movilidad laboral y de rentas en el impacto de la evolución del ciclo económico sobre el bienestar de las familias.

\section{Bibliografía}

Albert, C. y Toharia, L. (2001): Transiciones laborales en la UE. En Toharia, L. y L. Garrido (eds.): Análisis de las Condiciones de Vida a partir 
de la Encuesta Panel de Hogares de la Unión Europea (primer y segundo ciclos), Instituto Nacional de Estadística, Madrid.

Bentolila, S., Bover, O. y Arellano, M. (2002): Unemployment duration, benefit duration, and the business cycle, Economic Journal 112, 2002.

Bentolila, S., Bover, O. y Arellano, M. (2004): Paro y prestaciones: Nuevos resultados para España, in J. Pérez, C. Sebastián y P. Tedde (eds.), Estudios en Homenaje a Luis Ángel Rojo (Vol. I), Políticas, mercados e instituciones económicas, Madrid: Editorial Complutense.

Cantó, O. y Toharia, L. (2003): Las prestaciones por desempleo en España. Hacienda Pública Española, 3, 125-156.

Cebrián, I., Garrido, L. y Toharia, L. (1992): Los parados de larga duración y la protección social, en Luis Moreno y Manuel Perez Yruela, (comps.), Política social y estado del bienestar, Madrid, Ministerio de Asuntos Sociales, 351-377.

Costas, A. y Bel, G. (editores) (1997): Los beneficios de la liberalización: lecciones para España. Colección Estudios Económicos. La Caixa.

Espina, A., Fina, Ll., Sáez, F. y Toharia, L. (1984): La distribución funcional de la renta en España, Cuadernos del IMPI, 9, 22-32.

Fernández, F., Muro, J. y Toharia, L. (1988): El mercado de trabajo en España en 1987, Madrid, Fundación IESA.

García, I. y Toharia, L. (1998): Paro, pobreza y desigualdad en España: análisis transversal y longitudinal, Ekonomiaz, 40, 134-165.

Garrido, L. y Toharia, L. (1996): Paro y desigualdad, en Pobreza, necesidad y discriminación, II Simposio sobre Igualdad y Distribución de la Renta, Madrid, Fundación Argentaria, coleccion Igualdad, vol. 5, 9-63.

García Serrano, C., Malo, M. A. y Toharia, L. (1999): Desempleo y pobreza: un enfoque dinámico con ilustraciones del caso español, Revista de Occidente, 47-61.

García Serrano, C., Malo, M. A. y Toharia, L. (2001): La Pobreza en España: un Análisis Crítico Basado en el Panel de Hogares de la Unión Europea, Madrid, Ministerio de Trabajo y Asuntos Sociales. 
Muro, J., Raymond, J.L., Toharia, L. y Uriel, E. (1988a): Análisis de las condiciones de vida y trabajo en España, Madrid, Ministerio de Economía y Hacienda.

Muro, J., Raymond, J.L., Toharia, L. y Uriel, E. (1988b): La Encuesta de Población Activa y la Encuesta de Condiciones de Vida y Trabajo, Estadística Española, 119, 379-406.

Toharia, L. (1978): Interpersonal welfare comparisons and welfare economics: a comment, Kyklos, 31 (2), 311-314.

Toharia, L. (1984): La política de rentas en España, Cuadernos IMPI, 10, 4-15.

Toharia, L. (1997): El sistema español de protección por desempleo, Papeles de economía española, 72, 192-213.

Toharia, L. (2003): El mercado de trabajo en España 1978-2003, Información Comercial Española, 811, 203-220.

Toharia, L., Albert, C., García -Serrano, C., Malo, M. A., Davia, M. A. y Arranz, J. M. (2007): Empleo e inclusión social, Madrid, Ministerio de Trabajo y Asuntos Sociales.

Toharia, L. y Garrido, L. (2001): Análisis de las Condiciones de Vida a partir de la Encuesta Panel de Hogares de la Unión Europea (primer y segundo ciclos), Madrid, Instituto Nacional de Estadística.

Viñals, J. y Jimeno, J.F. (1997): El mercado de trabajo español y la unión económica y monetaria europea. Papeles de Economía Española, 72, 21-36. 
Cantó \& García Maínar / Revista de Economía Laboral 10 (2013), 48-68

Cuadro 1. Publicaciones sobre bienestar, condiciones de vida de las familias y mercado de trabajo

\begin{tabular}{|c|c|c|c|c|c|c|}
\hline Artículo & Coautores & Año & Publicación & Datos & Objetivo & Conclusiones \\
\hline $\begin{array}{l}\text { Interpersonal welfare } \\
\text { comparisons and welfare } \\
\text { economics: a comment }\end{array}$ & & 1978 & Kyklos & -- & $\begin{array}{l}\text { Comentario a un artículo de J.L. } \\
\text { Simons sobre distribución } \\
\text { óptima de la renta con } \\
\text { comparaciones interpersonales } \\
\text { de utilidad }\end{array}$ & $\begin{array}{l}\text { Los juicios de valor no explicitados por el autor en la Función de Bienestar } \\
\text { Social hacen el análisis irrelevante y la única relevancia del artículo tiene que } \\
\text { ver con un test al marco teórico de Bentham en sentido empírico sobre la } \\
\text { utilidad marginal decreciente de la renta. }\end{array}$ \\
\hline $\begin{array}{l}\text { La política de rentas en } \\
\text { España }\end{array}$ & & 1984 & Cuadernos IMPI & -- & $\begin{array}{l}\text { Análisis del crecimiento de las } \\
\text { rentas salariales entre } 1965 \text { y } \\
1984\end{array}$ & $\begin{array}{l}\text { El régimen franquista estableció un sistema fuertemente intervencionista de } \\
\text { la Administración en los niveles retributivos, especialmente en los } 40 \text { y } 50 \\
\text { mientras que los Acuerdos económicos y sociales entre gobierno, sindicatos y } \\
\text { patronal tras la transición tratan de moderar los salarios. }\end{array}$ \\
\hline $\begin{array}{l}\text { La distribución funcional de } \\
\text { la renta en España }\end{array}$ & $\begin{array}{l}\text { Espina, A., Fina, } \\
\text { Ll., Sáez, F. }\end{array}$ & 1984 & $\begin{array}{l}\text { Cuadernos del } \\
\text { IMPI }\end{array}$ & -- & $\begin{array}{l}\text { Análisis de la distribución de la } \\
\text { renta por funciones }\end{array}$ & $\begin{array}{l}\text { Durante el franquismo la Administración era fuertemente intervencionista en } \\
\text { cuanto a los niveles retributivos de las empresas y, además, los acuerdos } \\
\text { económicos y sociales tras la transición tienen como uno de sus principales } \\
\text { objetivos promover la moderación salarial. }\end{array}$ \\
\hline $\begin{array}{l}\text { Análisis de las condiciones de } \\
\text { vida y trabajo en España }\end{array}$ & $\begin{array}{l}\text { Juan Muro, Jose } \\
\text { Luis Raymond y } \\
\text { Ezequiel Uriel }\end{array}$ & 1988 & $\begin{array}{l}\text { Ministerio de } \\
\text { Economía y } \\
\text { Hacienda }\end{array}$ & ECVT & $\begin{array}{l}\text { Análisis del mercado laboral } \\
\text { para el diseño de políticas } \\
\text { económicas y sociales }\end{array}$ & $\begin{array}{l}\text { El mercado de trabajo español se puede descomponer en tres grupos: uno con } \\
\text { buenas condiciones laborales, buenos salarios y estabilidad elevada ( } 50 \% \text {, } \\
\text { otro caracterizado por justo lo contrario }(25 \%) \text { y un tercero intermedio }(25 \%) \text {. }\end{array}$ \\
\hline $\begin{array}{l}\text { La Encuesta de Población } \\
\text { Activa y la Encuesta de } \\
\text { Condiciones de Vida y Trabajo }\end{array}$ & $\begin{array}{l}\text { Juan Muro, Jose } \\
\text { Luis Raymond y } \\
\text { Ezequiel Uriel }\end{array}$ & 1988 & $\begin{array}{l}\text { Estadística } \\
\text { Española }\end{array}$ & EPA y ECVT & $\begin{array}{l}\text { Estudio comparativo } \\
\text { clasificaciones población en } \\
\text { relación con la actividad } \\
\text { económica en ambas fuentes }\end{array}$ & $\begin{array}{l}\text { La ECVT permite conocer ciertas actividades marginales del contingente de } \\
\text { parados. En cambio, la tasa de actividad laboral de la ECVT resulta ser } \\
\text { similar a la que se obtiene en la EPA. }\end{array}$ \\
\hline Paro y desigualdad & L. Garrido & 1996 & $\begin{array}{l}\text { Pobreza, Necesidad } \\
\text { y Discriminación (II } \\
\text { Simposio sobre } \\
\text { Igualdad y } \\
\text { Distribución de la } \\
\text { Renta) }\end{array}$ & EPA & $\begin{array}{l}\text { Analizar la incidencia del } \\
\text { desempleo (individual y familiar) } \\
\text { desde } 1977 \text { hasta } 1994 \text { para } \\
\text { explicar por qué la sociedad } \\
\text { española puede soportar una } \\
\text { tasa de paro tan alta sin sufrir } \\
\text { grandes tensiones sociales }\end{array}$ & $\begin{array}{l}\text { Una gran parte del alto número de desempleados de las crisis de los } 80 \text { y } 90 \\
\text { han podido sobrellevar la situación gracias al apoyo familiar, que ha } \\
\text { funcionado como un mecanismo redistribuidor de rentas que ha evitado que se } \\
\text { hayan dado tensiones sociales significativas. }\end{array}$ \\
\hline
\end{tabular}


Cuadro 2. Publicaciones sobre pobreza y protección social por desempleo

\begin{tabular}{|c|c|c|c|c|c|c|}
\hline Artículo & Coautores & Año & Publicación & Datos & Objetivo & Conclusiones \\
\hline $\begin{array}{l}\text { Los parados de larga } \\
\text { duración y la } \\
\text { protección social }\end{array}$ & $\begin{array}{l}\text { I. Cebrián y L. } \\
\text { Garrido }\end{array}$ & 1992 & $\begin{array}{l}\text { Política Social } \\
\text { y Estado de } \\
\text { Bienestar }\end{array}$ & EPA & $\begin{array}{l}\text { Análisis de parados de } \\
\text { larga duración. Bolsa } \\
\text { permanente o movilidad } \\
\text { en el colectivo }\end{array}$ & $\begin{array}{l}\text { El paro de larga duración no conlleva necesariamente la } \\
\text { permanencia en esta situación. Importante movilidad. } \\
\text { Algunos colectivos sí tienen problemas por falta de empleo y } \\
\text { carencia de protección social. }\end{array}$ \\
\hline $\begin{array}{l}\text { El sistema español de } \\
\text { protección por } \\
\text { desempleo }\end{array}$ & & 1997 & $\begin{array}{l}\text { Papeles de } \\
\text { Economía } \\
\text { Española }\end{array}$ & EPA, INEM & $\begin{array}{l}\text { Descripción del sistema } \\
\text { español de protección por } \\
\text { desempleo }\end{array}$ & $\begin{array}{l}\text { El sistema español de protección por desempleo ha } \\
\text { experimentado cambios en su extensión y aplicación, } \\
\text { encontrándose desde } 1992 \text { en niveles equiparables a los } \\
\text { demás países europeos. }\end{array}$ \\
\hline $\begin{array}{l}\text { Las prestaciones por } \\
\text { desempleo en España: } \\
\text { eficiencia y equidad }\end{array}$ & O. Cantó & 2003 & $\begin{array}{l}\text { Hacienda } \\
\text { Pública } \\
\text { Española }\end{array}$ & $\begin{array}{l}\text { EPA, INEM, } \\
\text { OCDE, } \\
\text { Comisión } \\
\text { Europea }\end{array}$ & $\begin{array}{l}\text { Evaluación de la } \\
\text { eficiencia y equidad del } \\
\text { sistema de protección por } \\
\text { desempleo }\end{array}$ & $\begin{array}{l}\text { Es necesario medir mejor la cobertura del sistema de } \\
\text { protección por desempleo, las prestaciones por desempleo } \\
\text { inhiben la búsqueda de empleo, e inciden negativamente en } \\
\text { la tasa de salida hacia el empleo a corto plazo y el sistema } \\
\text { español de protección por desempleo está en un nivel } \\
\text { intermedio en los países de la OCDE en cuanto al acceso, la } \\
\text { tasa de sustitución y la duración de las prestaciones. }\end{array}$ \\
\hline
\end{tabular}


Cuadro 3. Publicaciones sobre la dinámica del mercado de trabajo y la renta de las familias españolas

\begin{tabular}{|c|c|c|c|c|c|c|}
\hline Artículo & Coautores & Año & Publicación & Datos & Objetivo & Conclusiones \\
\hline $\begin{array}{l}\text { Los parados de larga duración } \\
\text { y la protección social }\end{array}$ & $\begin{array}{l}\text { I. Cebrián y L. } \\
\text { Garrido }\end{array}$ & 1992 & $\begin{array}{l}\text { Política Social y } \\
\text { Estado de } \\
\text { Bienestar }\end{array}$ & EPA & $\begin{array}{l}\text { Análisis de parados de larga } \\
\text { duración. Bolsa permanente o } \\
\text { movilidad en el colectivo }\end{array}$ & $\begin{array}{l}\text { El paro de larga duración no conlleva necesariamente la permanencia en esta } \\
\text { situación. Importante movilidad. Algunos colectivos sí tienen problemas por } \\
\text { falta de empleo y carencia de protección social. }\end{array}$ \\
\hline $\begin{array}{l}\text { Paro, pobreza y desigualdad } \\
\text { en España }\end{array}$ & I. García & 1998 & Ekonomiaz & UE y EPA & $\begin{array}{l}\text { Relación entre paro y pobreza e } \\
\text { importancia de la familia }\end{array}$ & $\begin{array}{l}\text { Clara relación paro-pobreza, sobre todo cuando nadie trabaja en la familia. } \\
\text { Más probabilidad de pobreza y de permanencia en mujeres con estudios bajos. } \\
\text { Gran movilidad en el mercado de trabajo. }\end{array}$ \\
\hline $\begin{array}{l}\text { Desempleo y pobreza: un } \\
\text { enfoque dinámico }\end{array}$ & $\begin{array}{l}\text { C. García-Serrano } \\
\text { y M. A. Malo }\end{array}$ & 1999 & $\begin{array}{l}\text { Revista de } \\
\text { Occidente }\end{array}$ & PHOGUE & Relación paro y pobreza & $\begin{array}{l}\text { La pérdida de empleo está relacionada con la pobreza pero no es la más } \\
\text { importante de las causas de la pobreza aunque la persistencia de la pobreza } \\
\text { está muy relacionada con la permanencia en el desempleo. Carácter muy } \\
\text { complejo de la relación paro-pobreza. }\end{array}$ \\
\hline $\begin{array}{l}\text { Transiciones laborales en la } \\
\text { UE }\end{array}$ & C. Albert & 2001 & $\begin{array}{l}\text { Análisis de las } \\
\text { condiciones de vida } \\
\text { a partir de la } \\
\text { encuesta PHOGUE }\end{array}$ & $\begin{array}{l}\text { PHOGUE (2 } \\
\text { primeras olas) }\end{array}$ & $\begin{array}{l}\text { Análisis de las trayectorias de } \\
\text { actividad a lo largo de } 24 \text { meses. }\end{array}$ & $\begin{array}{l}\text { Los datos del PHOGUE permiten realizar el análisis de las trayectorias de } \\
\text { actividad de los individuos a lo largo del tiempo distinguiendo entre ocupados, } \\
\text { parados e inactivos, y estudiando, por lo tanto las seis posibles transiciones. } \\
\text { También resulta interesante ampliar el análisis a los ocupados que trabajan } \\
\text { más de } 15 \text { horas y la utilización de índices agregados de movilidad. }\end{array}$ \\
\hline $\begin{array}{l}\text { La pobreza en España: un } \\
\text { análisis crítico basado en el } \\
\text { PHOGUE }\end{array}$ & $\begin{array}{l}\text { C. García-Serrano } \\
\text { y M. A. Malo }\end{array}$ & 2001 & $\begin{array}{l}\text { La pobreza en } \\
\text { España: un análisis } \\
\text { crítico basado en el } \\
\text { PHOGUE }\end{array}$ & PHOGUE & $\begin{array}{l}\text { Importancia de la pobreza } \\
\text { permanente en los países UE }\end{array}$ & $\begin{array}{l}\text { Importante grado de movilidad de entrada y salida de la pobreza. Alta } \\
\text { relación en la pobreza permanente de los que llevan mucho tiempo en el } \\
\text { desempleo. Similitud entre España y UE en este aspecto. Sin embargo, en } \\
\text { España, Grecia y Portugal el riesgo de ser pobre permanente está menos } \\
\text { concentrado según el historial laboral. }\end{array}$ \\
\hline $\begin{array}{l}\text { Análisis de la pobreza } \\
\text { persistente }\end{array}$ & $\begin{array}{l}\text { C. Albert, C. } \\
\text { García-Serrano, M. } \\
\text { A. Malo, M. A. } \\
\text { Davia y J. M. } \\
\text { Arranz }\end{array}$ & 2007 & $\begin{array}{l}\text { Empleo e Inclusión } \\
\text { Social }\end{array}$ & PHOGUE & $\begin{array}{l}\text { Estudio de la persistencia de la } \\
\text { pobreza }\end{array}$ & $\begin{array}{l}\text { La pobreza persistente y la ocasional obedecen a procesos parecidos y, por lo } \\
\text { tanto, las políticas de actuación deberían ser parecidas, si bien matizando la } \\
\text { intensidad de las políticas en función de ciertas variables, pues el tamaño de } \\
\text { la influencia de las variables relevantes es diferente. }\end{array}$ \\
\hline
\end{tabular}

